\title{
Magnetic Properties of Fe-Cu-Nb-Si-B Nanocrystalline Magnetic alloys
}

\author{
M. Garcia del Muro, X. Batlle, R. Zquiak and J. Tejada. \\ Departament de Fisica Fonamental, Universitat de Barcelona \\ Diagonal 647, 08028 Barcelona, Spain. \\ C. Polak, R. Grössinger \\ Institut für Experimental Physik, Technische Universität \\ Vienna, Austria.
}

\begin{abstract}
Several ribbons of composition $\mathrm{Fe}_{3.5} \mathrm{Cu}_{1} \mathrm{Nb}_{3} \mathrm{Si}_{16.5} \mathrm{~B}_{6}$ and $\mathrm{Fe}_{73} \mathrm{Cu}_{1} \mathrm{Nb}_{3} \mathrm{Si}_{13} \mathrm{~B}_{9}$ were prepared by annealing the as-quenched samples between $525^{\circ} \mathrm{C}$ and $700^{\circ} \mathrm{C}$, which induced nucleation of nanocrystallites of Fe hcc-type composition. Mean grain sizes were obtained from X-ray diffractiou. Static magnetic properties were measured with both a Magnet Plysik Hysteresis-Graph (up to $200 \mathrm{Oe}$ ) and a SIIE S.Q.U.I.D. Magnetometer (up to $50 \mathrm{kOe}$ ). Soft magnetic parameters (coercive field and initial permeability) were very sensitive to grain size. The ZFC magnetization at low field showed a broad peak at a temperature $T_{M}$, thus signalling a certain distribution of nanocrystalline sizes, and $T_{M}$ strongly decreased when the mean grain size decreased. Isothermal magnetization curves at low temperature showed the expected asymptotic behavior of a random magnet material at low and high fields.
\end{abstract}

\section{INTRODUCTION}

Recently, Yoshizawa et al. [1] reported on new ferromagnetic Fe-base alloys with ultrafine grain structure, exhibiting excellent soft magnetic properties. The material is as-cast as an amorphous ribbon. A subsequent heat treatment above the crystallization temperature produces the appearance of an ultrafine grain structure of $\alpha-\mathrm{FeSi}$ embedded in a disordered matrix. Typical grain size diameter of the Fe-Si phase is $D \approx 10 \mathrm{~nm}$. The formation of this nanocrystalline structure is ascribed to the addition of $\mathrm{Cu}$ and $\mathrm{Nb}$, due to the enhancement of the inhomogeneity and instability of the amorphous structure.

We present in this paper a study of the dependence on grain size of some physical properties of these new $\mathrm{Fe}-\mathrm{Cu}-\mathrm{Nb}-\mathrm{Si}-\mathrm{B}$. We have chosen two different compositions, with a fixed doping rate of $\mathrm{Cu}$ and $\mathrm{Nb}$ (the optimum one concerning the soft magnetic properties) and we have varied the $\mathrm{Si}$ and $\mathrm{B}$ contents, which strongly affects the crystallization behavior.

Finally, we study the law of approach to saturation. The asymptotic behavior of the isothermal magnetization curves $\mathrm{M}(\mathrm{H})$ at low and high fields are explained in the framework of the random anisotropy model [2].

\section{EXPERIMENTAL}

Amorphous ribbons of the composition $\mathrm{Fe}_{73.5} \mathrm{Cu}_{1} \mathrm{Nb}_{3} \mathrm{Si}_{16.5} \mathrm{~B}_{6}$ (sample $\mathrm{A}$ ) and $\mathrm{Fe}_{73.5} \mathrm{Cu}_{1} \mathrm{Nb}_{3} \mathrm{Si}_{13.5} \mathrm{~B}_{9}$ (sample B) were supplied by VAC. They were produced by rapid quenching from melt. The as-quenched samples were annealed at different temperatures between $525^{\circ} \mathrm{C}$ and $700^{\circ} \mathrm{C}$, in order to obtain different crystallization stages. The heat treatments were Manuscript received May 31,1993. performed in a conventional furnace under Ar atmosphere, and the annealing time was one hour in all the cases. X-ray diffraction patterns were recorded at room temperature using the $\mathrm{Cu} \mathrm{K} \alpha$ radiation with a graphite monocromator. Magnetic flux density measurements were carried out with a Magnet Physik Hysteresis-Graph (maximum applied field of $200 \mathrm{Oe}$ ). Magnetization measurements were recorded with a SHE S.Q.U.I.D. magnetometer in the temperature range $6-300 \mathrm{~K}$ and magnetic fields up to $50 \mathrm{kOe}$. The zero-field-cooled (ZFC) and field-cooled (FC) processes were measured at low fields (at about $25 \mathrm{Oe}$ ).

\section{RESULTS AND DISCUSSION}

\section{A. X-ray Diffraction}

$\mathrm{X}$-ray diffraction patterns of the annealed samples show a bcc peak. The average grain size was calculated from the grain size distribution (see Fig.1), and the line profile analysis reached the proofed sensitivity at a minimum grain size of about $25 \mathrm{~nm}$, which corresponds to the grain size of the gold reference sample. Therefore, the smallest values indicate only that the grain size is much smaller than $25 \mathrm{~nm}$.

It is observed that the size of the $\alpha-\mathrm{Fe}(\mathrm{Si})$ solid solution increases with the annealing temperature (see Table I), and the recrystallization behavior depends on the $\mathrm{Si}$ and $\mathrm{B}$ contents, as previously noted by Polak et al.[3].

\section{B. Soft Magnetic Properties}

The coercive field $H_{c}$ and the initial permeability $\mu_{i}$ (see Table I) have been obtained at room temperature from the dependence of the magnetic flux density on the applied field $\left(\mathrm{H}_{\max }=200 \mathrm{Oe}\right)$. It is shown that above a critical annealing temperature (and consequently, above a critical grain size) $\mathrm{H}_{c}$ sharply increases and $\mu_{\mathrm{i}}$ decreases. This critical temperature is about $600-625^{\circ} \mathrm{C}$ and the critical grain size is about $30 \mathrm{~nm}$ for both series of samples. The observed behavior is in agreement with the theoretical results obtained by Herzer [4], assuming the random anisotropy model developed by Alben et al. [2]. 
TABLE I

GS: average grain size.

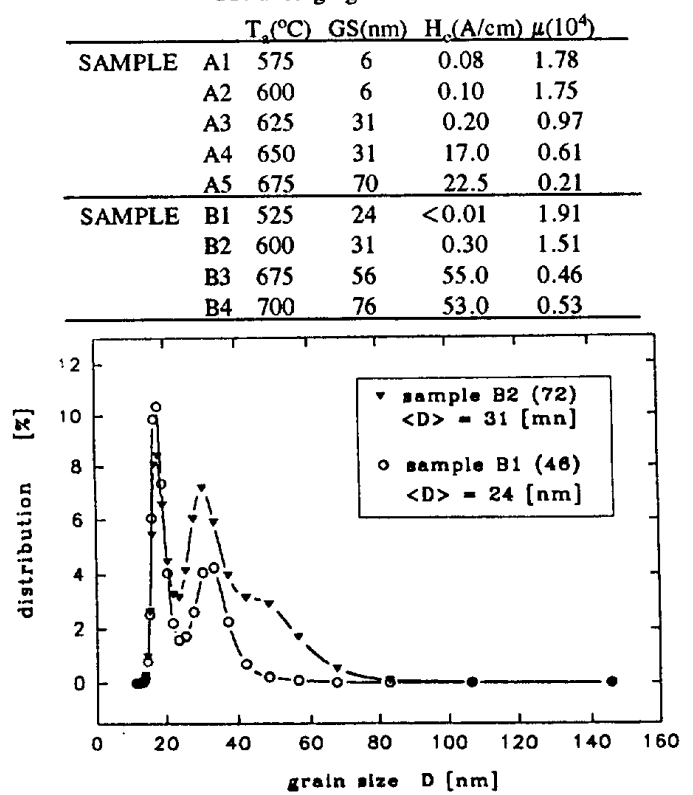

Fig.1. Distribution in $\%$ of the grain size of the sample B1 and B2:the average grain size was calculated using this distribution. The first peak corresponds to the reference sample (gold).

\section{ZFC-FC Magnetization}

We show in Fig. 2a, 2b, 2c the ZFC and FC magnetization data for three samples of the A-type compound: A1 $\left(T_{a}=575^{\circ} \mathrm{C}\right.$, grain size $\left.=6 n m\right), A 4 \quad\left(T_{a}=650^{\circ} \mathrm{C}\right.$, grain size $=31 \mathrm{~nm})$ and $A 5\left(\mathrm{~T}_{\mathrm{a}}=675^{\circ} \mathrm{C}\right.$, grain size $\left.=70 \mathrm{~nm}\right)$. The different behavior of the ZFC and FC curves is a typical feature of a magnetic system in which energy levels do not only depend, at a given temperature, on the Boltzman distribution but it is also strongly afected by the thermomagnetic story. This magnetic behavior is also observed, for example, in spin glasses, small particle systems, ultrafine grains embedded in an amorphous matrix and clusters of atoms.

In our present case, the $\mathrm{ZFC}$ curves show a very wide maximum, indicating that we have a certain distribution of blocking temperatures (because of grain sizes) for each sample. The strong decrease of this average blocking temperature in the samples follows the strong reduction of the mean grain size. Moreover, the ZFC magnetization decreases below the peak (where the magnetic moment of each grain is blocked), showing the randomness of the easy magnetization direction of each grain. There also appears a clear irreversibility between the ZFC and FC measurements. The $\mathrm{FC}$ magnetization is always above the $\mathrm{ZFC}$ values since, in the former process, the magnetic moments of the grains tend to block along the magnetic field axis below a certain temperature (maximum of the FC magnetization).
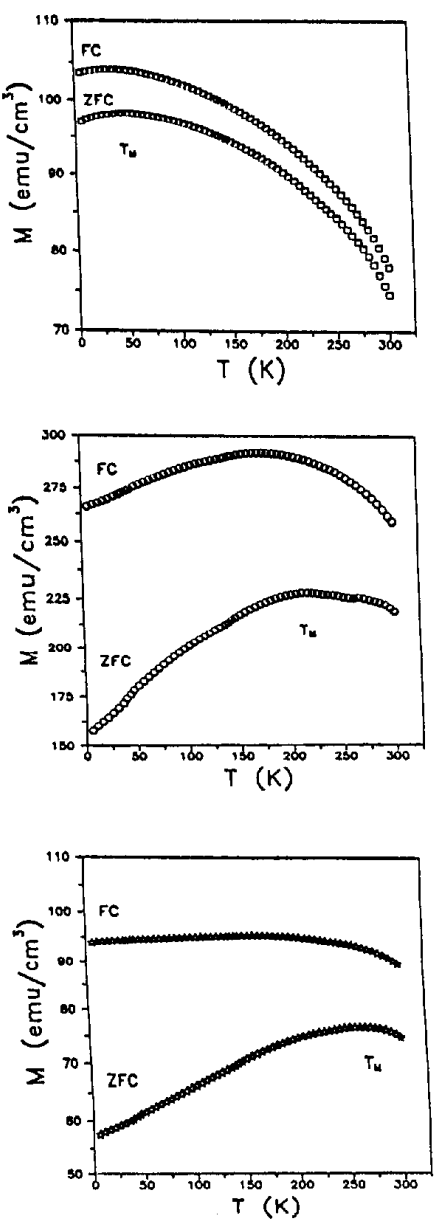

Fig.2 ZFC and FC magnetization of three selected samples of the A-type compound. The maximum of the $\mathrm{ZFC}$ is observed at $T_{M} \approx 50 \mathrm{~K}$ for sample $A 1(a), T_{M} \approx 210 \mathrm{~K}$ for sample $A 4(b)$ and $T_{M} \approx 262 \mathrm{~K}$ for A5(c).

\section{Isothermal Magnetization Curves}

The isothermal magnetization curves $\mathrm{M}(\mathrm{H})$ were measured at $6 \mathrm{~K}$ for samples $\mathrm{A} 1, \mathrm{~A} 4$ and $\mathrm{A} 5$, with a maximum applied field of $50 \mathrm{kOe}$.

We may explain the behavior of the magnetization as we approach saturation in the framework of the random anisotropy model, applied to amorphous ferromagnets [5].

This model assumes that the local anisotropy axes are correlated along a certain distance $R_{a}$ and their spatial distribution is characterized by a certain distribution function $\mathrm{C}(\mathrm{x})$ which reflects short range crystallographic order. Further, the magnetic behavior of a disordered system depends on the ratio between the random anisotropy field $\mathrm{H}_{\mathrm{r}}$ $=2 \mathrm{~K}_{\mathrm{r}} / \mathrm{M}_{\mathrm{o}}$ (where $\mathrm{K}_{\mathrm{r}}$ is the anisotropy constant) and the exchange field $H_{e x}=2 A /\left(R_{2}{ }^{2} M_{0}\right)$ (where $A$ is the exchange strength). If we define the crossover parameter [5] $\lambda_{r}$, as 
$\lambda_{\mathrm{r}}=(2 / 15)^{1 / 2}\left(\mathrm{H}_{\mathrm{r}} / \mathrm{H}_{\mathrm{cx}}\right)$, the low anisotropy regime corresponds to $\lambda_{\mathrm{r}}<1$, when the ferromagnetic correlation length, defined as $R_{f}=R_{a} / \lambda_{r}{ }^{2}$ is larger than $R_{a}[6]$, and it would lead to long range magnetic order. However, although ferromagnetic clusters of spins are formed at small distances, long range ferromagnetic order is destroyed at large distances due to random fields which cause weak fluctuations of the spin orientation, as it was noticed by Imry and Ma [7]. Assuming that there is no coherent anisotropy in our system, a correlated spin glass (CSG) state is attained [6]: magnetization smoothly and stochastically rotates over the solid. Furthermore, a partial order is induced in the solid when a magnetic field is applied. In this case, the magnetization vectors wander as one moves about the system due to random fields and the CSG state gives place to a ferromagnet with wandering axes state (FWA). For the latter, Chudnovsky et al. [5] showed that the magnetization law should follow a $\mathrm{H}^{-1 / 2}$ dependence when $\mathrm{H}<<\mathrm{H}_{\mathrm{ex}}$ and $\mathrm{a} \mathrm{H}^{-2}$ dependence when $\mathrm{H}$ $>>\mathrm{H}_{\mathrm{ex}}$ regardless the form of $\mathrm{C}(\mathrm{x})$ :

$$
\begin{array}{ll}
\Delta M / M_{o}=\left(v_{c} / 30\right)\left(H_{r} / H_{e x}\right)^{2}\left(H_{e x} / H\right)^{1 / 2} & \text { if } H<<H_{e x} \\
\Delta M / M_{o}=(1 / 30)\left(H_{r} / H\right)^{2} & \text { if } H>>H_{e x}
\end{array}
$$

where $v_{c}=\int_{0}{ }^{\infty} d y \cdot y^{2} \cdot C(y)$

Two important aspects should be taken into account before applying eq. (1) and (2) to the study of the asymptotic behavior of the magnetization curves. The first concerns the inevitable presence of some coherent anisotropy in the sample. Although a weak coherent anisotropy, satisfying $\lambda_{c}{ }^{1 / 2}$ $>\lambda_{r}{ }^{2}$ (where $\lambda_{c}=(2 / 15)^{1 / 2}\left(H_{c} / H_{e x}\right)$, and $H_{c}$ is the coherent anisotropy field) destroys the CSG state [6], the effect of $H_{c}$ becomes unimportant when $\mathrm{H}>>\mathrm{H}_{\mathrm{c}^{*}}$. Secondly, the $\mathrm{H}_{\mathrm{ex}}$ must be accessible experimentally.

We have chosen samples A5 and A1 (the largest and smallest A samples) in order to test the validity of the low anisotropy limit of the random anisotropy model when applied to these $\mathrm{Fe}-\mathrm{Cu}-\mathrm{Nb}-\mathrm{Si}-\mathrm{B}$ magnetic alloys.

Concerning the A.5 sample, the linear dependence of $\Delta M$ with respect to $\mathrm{H}^{-1 / 2}$ and $\mathrm{H}^{-2}$ is shown in Fig.3: in Fig. 3a we show $M$ as a function of $\mathrm{H}^{-1 / 2}$, while in Fig. $3 b \log (\Delta M)$ versus $\log (H)$ is displayed, whence $\Delta M=M-M_{o}-\chi_{d} H$ and $\chi_{d}$ is the high field differential susceptibility. Equations (1) and (2) together with the experimental magnetization curve at $6 \mathrm{~K}$ enable us to estimate that $\mathrm{H}_{\mathrm{r}} \approx 4000 \mathrm{Oe}$ and $\mathrm{H}_{\mathrm{cx}} \approx 2100 \mathrm{Oe}$, thus leading to $\lambda_{r} \approx 0.7-0.8<1$. Besides, if we assume a typical value of the coherent anisotropy for these kinds of systems $\left(\mathrm{K}_{\mathrm{c}} \approx 10^{5} \mathrm{erg} / \mathrm{cm}^{3}[8]\right)$, we obtain $\lambda_{\mathrm{c}} \approx 0.03$, which implies $\lambda_{\mathrm{r}}{ }^{2}>\lambda_{\mathrm{c}}^{1 / 2}$. These results verify the self consistency of the model.

Concerning the Al sample (grain size $=6 \mathrm{~nm}$ ), a different asymptotic behavior is observed at low fields: $\Delta M \propto \mathrm{H}^{-1}$ (see Fig.4). This behavior, predicted theoretically by Chudnovsky [9] and Ruiz et al. [10] for a two-dimensional random
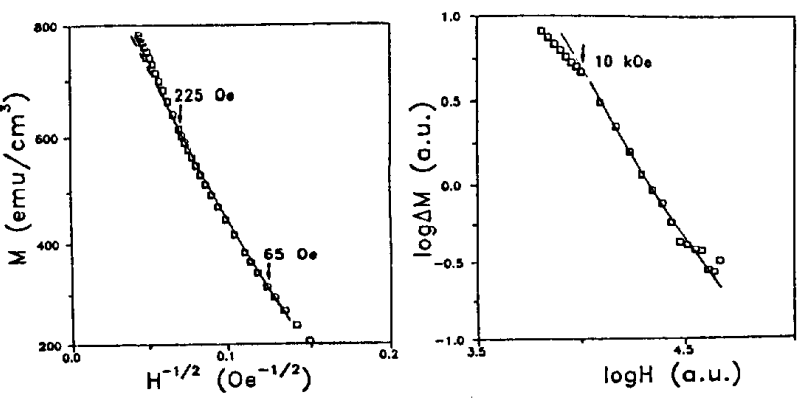

Fig.3 Two linear regimes of approach to saturation for sample A5 at $6 \mathrm{~K}$ : $M$ versus $\mathrm{H}^{-1 / 2}$ and $\log (\Delta \mathrm{M})$ versus $\log (\mathrm{H})$ (where the slope of the $\log$ - $\log$ curve is $\mathbf{m}=2$ ).

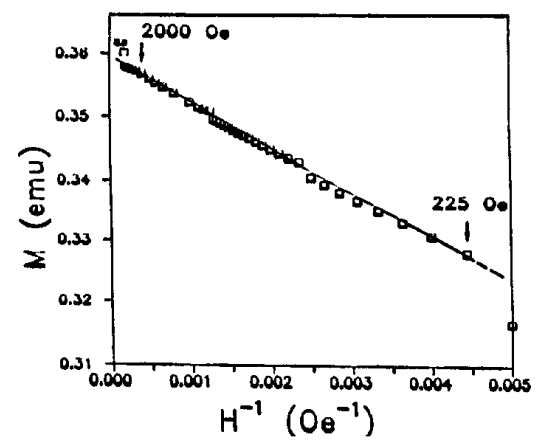

Fig.4 Linear regime for sample $A 1$ at low fields: $\mathrm{M}$ versus $\mathrm{H}^{-1}$.

anisotropy system in the low anisotropy limit, has been observed experimentally in magnetic multilayers [10]. In our case, the predicted asymptotic behavior at high fields $(\Delta M \propto$ $\mathrm{H}^{-2}$ ) is difficult to observe, since $\Delta \mathrm{M}$ becomes so small at large fields that weak effects (magnetism of conducting electrons, paramagnetic impurities, ...) violate this dependence.

\section{REFERENCES}

[1] Y. Yoshizawa, S. Oguma and K. Yamauchi, J.Appl.Phys., vol. 64, pp. $6044-6046,1988$.

[2] R. Alben, J.J. Becker and C. Chi, J.Appl.Phys., vol. 49, pp. 1653 $1658,1978$.

[3] C. Polak, R. Grössinger, H. Sassik and H. Herzer, J.Mag.Mag.Mat., vol. 100, pp.104 - 107, 1992.

[4] G. Herzer, IEEE Trans.Magn., vol. 25, pp. 3327 - 3329, 1989.

[5] E.M. Chudnovsky, W.M. Saslow and R.A. Serota, Phys.Rev.B, vol. 33, pp. $251-261,1986$.

[6] E.M. Chudnovsky, J.Appl.Phys., vol. 64, pp. 5770 - 5775, 1988.

[7] Y. Imri and S. Ma, Phys.Rev.Lett., vol. 35, pp. 1399 - 1401, 1975.

[8] R.W. Chantrel, J.Mag.Mag.Mat., vol. 95, pp. 365 - 378, 1991.

[9] E.M. Chudnovsky, J.Mag.Mag.Mat., vol. 40, pp. 21 - 26, 1983.

[10] J.M. Ruiz et al., Phys.Rev.B, vol. 47, in press. 